\title{
A spectropolarimetric survey of the coolest magnetic Ap stars
}

\author{
N. Johnson ${ }^{1}$, G.A. Wade ${ }^{1}$, M. Aurière ${ }^{2}$, J.-F. Donati ${ }^{2}$, F. Lignir̀res ${ }^{2}$, S. \\ Marsden $^{2}$, D. Mouillet ${ }^{2}$, F. Paletou ${ }^{2}$, N. Toqué ${ }^{2}$, P. Petit ${ }^{3}$, S. \\ Bagnulo $^{4}$, J.D. Landstreet ${ }^{5}$ and T. Lüftinger ${ }^{6}$ \\ ${ }^{1}$ Department of Physics, Royal Military College of Canada \\ ${ }^{2}$ Observatoire Midi Pyrénées, France \\ ${ }^{3}$ Max Planck Institut für Astrophysik, Germany \\ ${ }^{4}$ European Southern Observatory, Chile \\ ${ }^{5}$ University of Western Ontario, Canada \\ ${ }^{6}$ Universitat Wien Institut für Astronomie, Austria
}

\begin{abstract}
We describe the first results of a systematic spectropolarimetric survey of the coolest magnetic Ap stars, undertaken with the MuSiCoS spectropolarimeter, with the aim of clarifying the magnetic field and atmospheric characteristics of these enigmatic objects.
\end{abstract}

Keywords. Methods: data analysis, techniques: polarimetric, techniques: spectroscopic, stars: chemically peculiar, stars: magnetic fields

\section{Overview}

We are conducting a magnetic survey of all Northern classified Ap stars with spectral types later than A5. Observations are obtained using the MuSiCoS spectropolarimeter at Pic du Midi Observatory. The sample of about 90 stars was selected to include all observable cool Ap stars from the Bright Star Catalogue (5th revised edition, Hoffleit et al. 1995) and the General Catalogue of Ap and Am stars (Renson et al. 1991). These lists were supplemented with observable roAp stars. Multiple circular polarisation spectra are obtained for each target, and the spectroscopic properties and magnetic field characteristics are inferred. Detection and diagnosis of the magnetic field is obtained using Least-Squares Deconvolution (Donati et al. 1997).

\section{Motivation}

This study is meant to complement the search for weak magnetic fields in Ap stars, see Aurière \& Wade (2005). Whereas that study focuses primarily on hotter Ap/Bp stars, here we study exclusively the rarer cool Ap and Fp stars in an attempt to characterise their magnetic and spectroscopic properties. Because these stars straddle the effective temperatures at which surface convection becomes dynamically important in the outer layers of Main Sequence stars (around an effective temperature of $8000 \mathrm{~K}$ ), their magnetic fields and surface chemistry may contain unique information about the role of convective mixing on chemical separation and magnetic field evolution. The coolest Fp stars also display the most extreme chemical peculiarities. Some are also non-radial acoustic p-mode pulsators (roAp stars). The excitation mechanism of these waves is still not understood. RoAp stars represent one of the greatest mysteries amongst the A-type stars. 
LS deconvolved Zeeman signature (2615 lines used)

LS deconvolved Zeeman signature (2098 lines used)
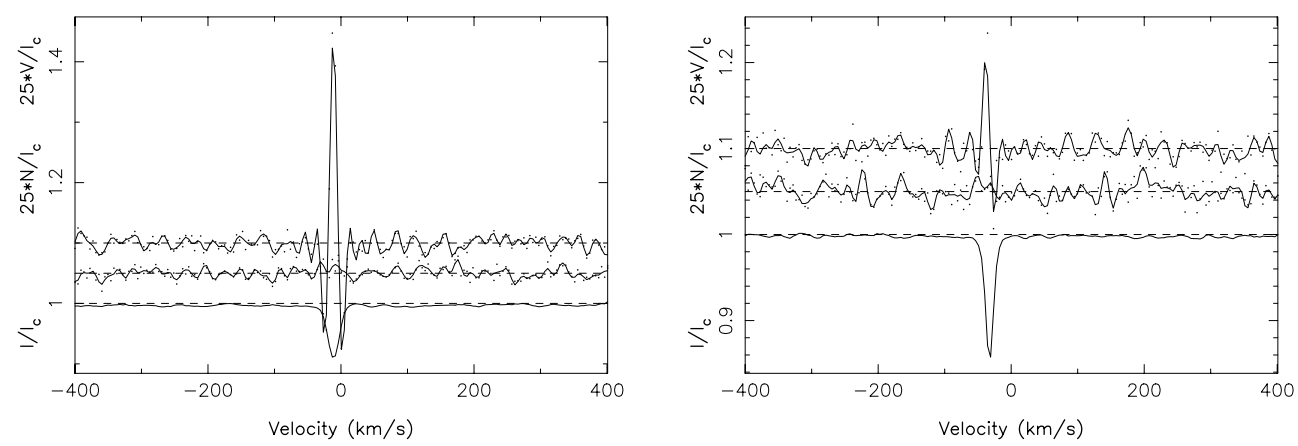

Figure 1. Left: The cool, poorly-studied Northern roAp star HD $12098\left(B_{z}=+19 \pm 80 \mathrm{G}\right)$

Right: The well-known roAp star HD $122970\left(B_{z}=+131 \pm 47 \mathrm{G}\right)$.

LS deconvolved Zeeman signature (2670 lines used)

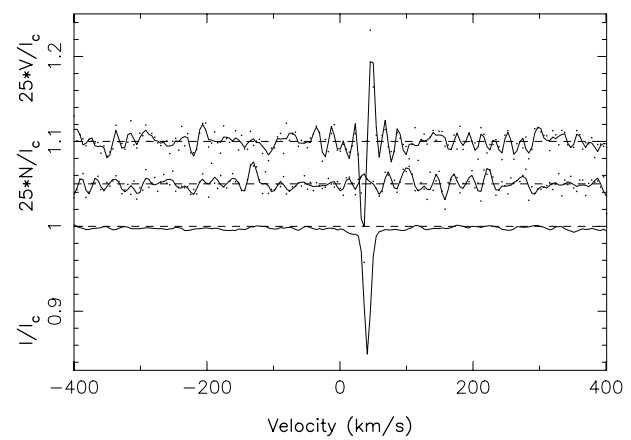

LS deconvolved Zeeman signature (2836 lines used)

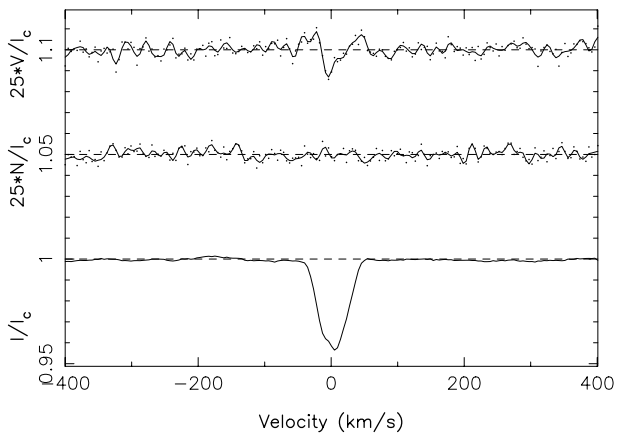

Figure 2. Left: A newly-detected magnetic Ap star, HD $138633\left(B_{z}=-246 \pm 48 \mathrm{G}\right)$ Right: A puzzling cool Ap star, HD $96707\left(B_{z}=+1 \pm 37 \mathrm{G}\right)$.

LS deconvolved Zeeman signature (2847 lines used)

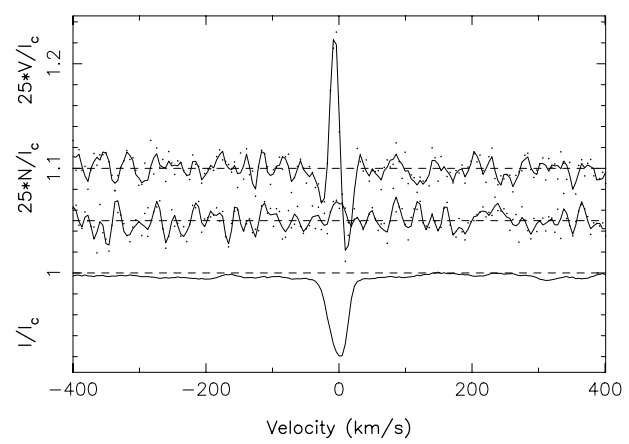

LS deconvolved Zeeman signature (1534 lines used)

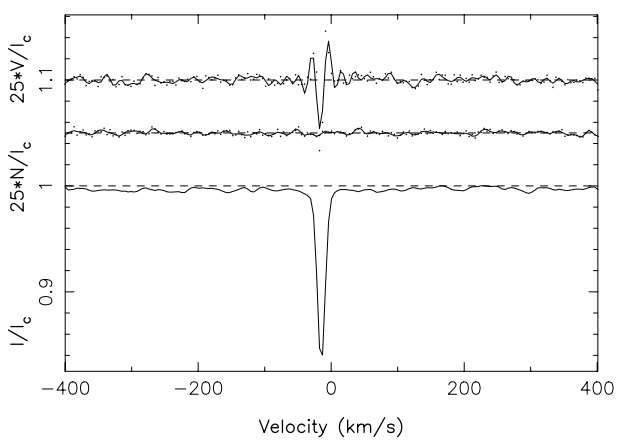

Figure 3. Two-newly-detected magnetic roAp stars: Left: HD $204117\left(B_{z}=+182 \pm 86 \mathrm{G}\right)$ Right: HD $204411\left(B_{z}=-30 \pm 11 \mathrm{G}\right)$.

\section{Results}

One hundred and eight observations of 36 classified cool Ap stars have been obtained during 3 observing runs during winter 2003, summer 2003, and winter 2004. For 6 stars, magnetic fields are detected for the first time. We show here LSD Stokes $I$ and $V$ mean profiles for 8 illustrative objects: 5 represent new magnetic field detections, 2 represent magnetic field detections in previously known magnetic stars, and 3 provide no evidence 

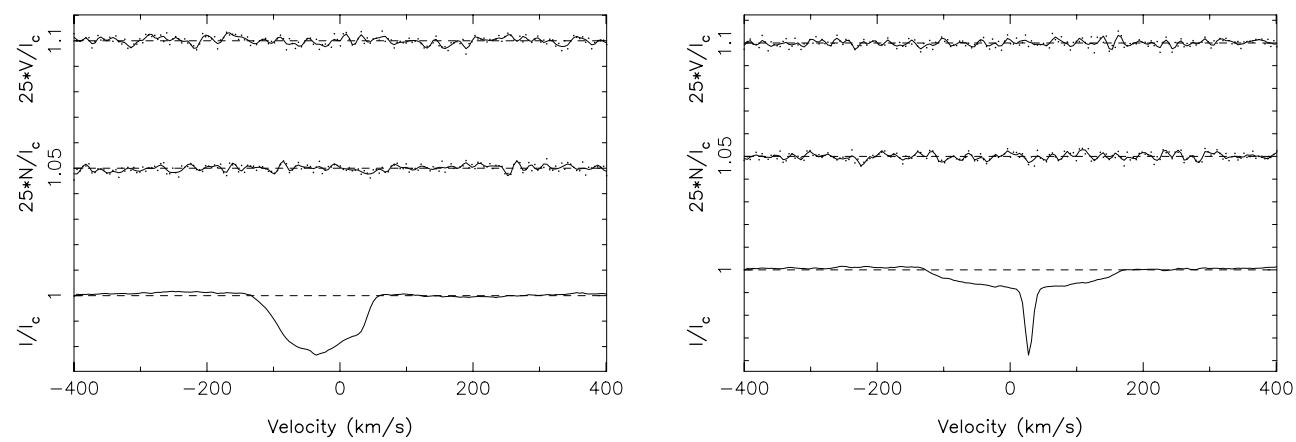

Figure 4. Two spectroscopically-classified Ap stars in which magnetic fields were not detected, Left: HD 182640 ( $\left.B_{z}=-60 \pm 41 \mathrm{G}\right)$ Right: HD 50635 ( $\left.B_{z}=-121 \pm 102 \mathrm{G}\right)$. Possibly misclassified?

Figure 5. Left: HD 22128, an apparently non-magnetic SB2 system, previously classified as Ap $\left(B_{z}=+32 \pm 49 \mathrm{G}\right)$ Right: HD 341037, a newly-detected magnetic Ap $\operatorname{star}\left(B_{z}=-820 \pm 167 \mathrm{G}\right)$.

for magnetic fields. Along with the mean Stokes $I$ and $V$ profiles, we also show the diagnostic $N$ (null) mean profile, and the inferred longitudinal magnetic field $B_{z}$ (note that the presence of a magnetic field is inferred from the detection of significant line circular polarisation, which may not necessarily correspond to a significant $B_{z}$ ). The identification and spectral type of each star is indicated at the top of each figure.

\section{Outlook}

This is an ongoing programme. Additional observations are currently being obtained at the Bernard Lyot telescope at Pic du Midi Observatory. We fully expect to complete the survey during February 2005. Preliminary results are reported by Johnson (2004).

\section{Acknowledgements}

The authors thank the Pic du Midi Observatory for generous allocations of observing time. GAW and JDL acknowledge support from the Natural Science and Engineering Research Council of Canada.

\section{References}

Aurière, M, Wade, G. 2005, These Proceedings, EP12

Donati, J.-F., Semel, M., Carter, B.D., Rees, D.E., Cameron, A.C., MNRAS, 291, 658-682, 1997

Hoffleit, D., Warren, W.H. Jr, CDS (The Bright Star Catalogue, $5^{\text {th }}$ revised edition), 1995

Johnson, N.M., M.Sc. thesis, Royal Military College of Canada, 2004

Renson, P., Gerbaldi, M.. Catalano, F. A., A\&A, 89, 429-433, 1991 